\title{
Two-point boundary value problems for linear evolution equations
}

\author{
Article \\ Published Version
}

Fokas, A. S. and Pelloni, B. (2001) Two-point boundary value problems for linear evolution equations. Mathematical Proceedings of the Cambridge Philosophical Society, 131 (3). pp. 521-543. ISSN 1469-8064 Available at https://centaur.reading.ac.uk/15684/

It is advisable to refer to the publisher's version if you intend to cite from the work. See Guidance on citing.

Published version at: http://journals.cambridge.org/action/displayAbstract? fromPage $=$ online $\&$ aid $=90385 \&$ fulltextType $=$ RA\&fileld $=S 0305004101005436$

Publisher: Cambridge University Press

All outputs in CentAUR are protected by Intellectual Property Rights law, including copyright law. Copyright and IPR is retained by the creators or other copyright holders. Terms and conditions for use of this material are defined in the End User Agreement.

www.reading.ac.uk/centaur

\section{CentAUR}

Central Archive at the University of Reading 
Reading's research outputs online 
Mathematical Proceedings of the Cambridge Philosophical Society

http://journals.cambridge.org/PSP

Additional services for Mathematical Proceedings of the Cambridge

Philosophical Society:

Email alerts: $\underline{\text { Click here }}$

Subscriptions: $\underline{\text { Click here }}$

Commercial reprints: $\underline{\text { Click here }}$

Terms of use : $\underline{\text { Click here }}$

\section{Two-point boundary value problems for linear evolution equations}

A. S. FOKAS and B. PELLONI

Mathematical Proceedings of the Cambridge Philosophical Society / Volume 131 / Issue 03 / November 2001, pp 521 - 543

DOI: DOI:10.1017/S0305004101005436, Published online: 26 November 2001

Link to this article: http://journals.cambridge.org/abstract_S0305004101005436

How to cite this article:

A. S. FOKAS and B. PELLONI (2001). Two-point boundary value problems for linear evolution equations. Mathematical Proceedings of the Cambridge Philosophical Society, 131, pp 521-543 doi:DOI:10.1017/S0305004101005436

Request Permissions : $\underline{\text { Click here }}$ 


\title{
Two-point boundary value problems for linear evolution equations
}

\author{
By A. S. FOKAS and B. PELLONI* \\ Department of Mathematics, Imperial College, London SW7 $2 B Z$. \\ e-mail: a.fokas@damtp.cam.ac.uk, b.pelloni@ic.ac.uk
}

(Received 20 January 2000; revised 28 February 2001)

\section{Abstract}

We study boundary value problems for a linear evolution equation with spatial derivatives of arbitrary order, on the domain $0<x<L, 0<t<T$, with $L$ and $T$ positive finite constants. We present a general method for identifying well-posed problems, as well as for constructing an explicit representation of the solution of such problems. This representation has explicit $x$ and $t$ dependence, and it consists of an integral in the $k$-complex plane and of a discrete sum. As illustrative examples we solve some two-point boundary value problems for the equations $i q_{t}+q_{x x}=0$ and $q_{t}+q_{x x x}=0$.

\section{Introduction}

A new method for solving boundary value problems for linear and for integrable nonlinear PDEs in two dimensions has been recently introduced (see review [4]). In this paper we apply this method, and in particular the results of [7], to solve twopoint boundary value problems for a general linear dispersive evolution equation. Let the scalar function $q(x, t)$ satisfy

$$
\left(\partial_{t}+i \sum_{j=0}^{n} \alpha_{j}\left(-i \partial_{x}\right)^{j}\right) q(x, t)=0, \quad 0<x<L, \quad 0<t<T,
$$

where $\alpha_{j}$ are real constants, $\alpha_{n} \neq 0$, and $L, T$ are positive constants. Let $q(x, t)$ also satisfy the initial condition

$$
q(x, 0)=q_{0}(x), \quad 0<x<L,
$$

where $q_{0}(x)$ is a sufficiently smooth function.

The dispersion relation of equation $(1 \cdot 1)$ is given by

$$
\omega(k)=\sum_{j=1}^{n} \alpha_{j} k^{j}
$$

The method we present identifies the set of boundary conditions that must be prescribed at $x=0$ and at $x=L$ in order for the resulting initial boundary value problem for equation $(1 \cdot 1)$ to be well posed. This method also yields the construction

\footnotetext{
* Current address: Department of Mathematics, University of Reading, Reading RG6 6AX, UK.
} 
of an explicit representation of the solution of such problems. This representation has explicit $x$ and $t$ dependence, and it consists of an integral in the $k$-complex plane, and of a discrete sum.

In order to describe the results proved in this paper,we define the following contours and domains in the $k$-complex plane.

Definition $1 \cdot 1$. Let $D, D_{+}$and $D_{-}$be the domains in the $k$-complex plane defined by

$$
D=\{k \in \mathbb{C}: \operatorname{Im} \omega(k)>0\}, \quad D_{+}=D \cap \mathbb{C}^{+}, \quad D_{-}=D \cap \mathbb{C}^{-},
$$

where $\mathbb{C}^{+}$and $\mathbb{C}^{-}$indicate the upper and lower half plane, respectively.

$$
\partial D_{+}, \partial D_{-} \text {are the oriented boundaries of } D_{+}, D_{-},
$$

where the orientation is such that $D$ is on the left-hand side of the increasing direction of $\partial D$.

It is shown in [7] that the components of $D$ are simply connected and unbounded, and that there exists an $R>0$ such that, outside the curve $|\omega(k)|=R, \partial D$ is the union of smooth disjoint simple contours that approach asymptotically, as $k \rightarrow \infty$, the rays of the variety $\operatorname{Im}(k+\alpha)^{n}=0$, where $\alpha=\alpha_{n-1} /\left(n \alpha_{n}\right)$. Moreover, the following lemma holds [7].

Lemma $1 \cdot 1$. Let $D_{R}$ be defined by

$$
D_{R}=\{k \in D:|\omega(k)|>R\} .
$$

Let $D_{R,+}$ and $D_{R,-}$ denote the part of $D_{R}$ in $\mathbb{C}^{+}$and $\mathbb{C}^{-}$respectively, i.e.

$$
D_{R,+}=D_{R} \cap \mathbb{C}^{+}, \quad D_{R,-}=D_{R} \cap \mathbb{C}^{-} .
$$

If $R$ is sufficiently large, $D_{R}$ has $n$ components, $D_{R,+}$ has $N$ components, and $D_{R,-}$ has $n-N$ components, where $N$ is given by

$$
N=\left\{\begin{array}{lll}
n / 2 & n \text { even }, & \\
(n+1) / 2 & n \text { odd }, & \alpha_{n}>0, \\
(n-1) / 2 & n \text { odd }, & \alpha_{n}<0 .
\end{array}\right.
$$

Definition $1 \cdot 2$. We denote by $D_{R, 1}, D_{R, 2}, \ldots, D_{R, N}$ the $N$ components of $D_{R,+}$. We denote by $D_{R, N+1}, D_{R, N+2}, \ldots, D_{R, n}$ the $n-N$ components of $D_{R,-}$.

The method discussed here has as its starting point the observation that any linear PDE in two dimensions, with constant coefficients, can be written as the condition that a certain differential 1 -form $w(x, t, k), k \in \mathbb{C}$, is closed. Its implementation involves the following three steps.

Step 1. Assume the existence of a solution $q(x, t)$ of $(1 \cdot 1)$, which is sufficiently smooth up to the boundary of the domain. By performing the spectral analysis of the 1 -form associated with equation $(1 \cdot 1)$ :

$(1 \cdot a)$ Construct an integral representation of the solution $q(x, t)$ in terms of certain functions of $k$ called the spectral functions. This is an immediate consequence of the closure of $w(x, t, k)$. For the case of equation $(1 \cdot 1)$, the spectral functions are defined in terms of $q_{0}(x)=q(x, 0), \partial_{x}^{j} q(0, t)$ and $\partial_{x}^{j} q(L, t), 0 \leqslant j \leqslant n-1$.

$(1 \cdot b)$ Obtain the global relation satisfied by the spectral functions. 
For equation (1·1), the implementation of this step yields (see Section 2):

Proposition 1.1 (Representation of solutions of equation (1.1)). Assume that $q(x, t)$ is a sufficiently smooth solution of $(1 \cdot 1)$. Then $q(x, t)$ is given by

$$
\begin{aligned}
q(x, t)= & \frac{1}{2 \pi}\left\{\int_{-\infty}^{\infty} e^{i k x-i \omega(k) t} \hat{q}_{0}(k) d k\right. \\
& \left.+\int_{\partial D_{+}} e^{i k x-i \omega(k) t} \hat{Q}(0, k) d k+\int_{\partial D_{-}} e^{i k(x-L)-i \omega(k) t} \hat{Q}(L, k) d k\right\},
\end{aligned}
$$

where

$$
\hat{q}_{0}(k)=\int_{0}^{L} e^{-i k x} q_{0}(x) d x, \quad k \in \mathbb{C},
$$

and the spectral functions $\hat{Q}(0, k), \hat{Q}(L, k)$ are defined by

$$
\begin{gathered}
\hat{Q}(\alpha, k)=\sum_{j=1}^{n} \alpha_{j}\left(\hat{Q}_{j-1}(\alpha, k)+k \hat{Q}_{j-2}(\alpha, k)+\cdots+k^{j-1} \hat{Q}_{0}(\alpha, k)\right), \\
\hat{Q}_{j}(\alpha, k)=\int_{0}^{T} e^{i \omega(k) t}\left(-i \partial_{x}\right)^{j} q(\alpha, t) d t, \quad j=0, \ldots, n-1, \quad k \in \mathbb{C},
\end{gathered}
$$

with $\alpha=0, L$.

Moreover, the boundary values of $q(x, t)$ satisfy the global relation

$$
\hat{Q}(0, k)-e^{-i k L} \hat{Q}(L, k)=-\hat{q}_{0}(k)+e^{i \omega(k) T} \hat{q}_{T}(k), \quad k \in \mathbb{C},
$$

where

$$
\hat{q}_{T}(k)=\int_{0}^{L} e^{-i k x} q(x, T) d x .
$$

Step 2. Given $q_{0}(x) \in \mathbf{C}^{\infty}[0, L]$, assume the existence of an admissible set of functions $\left\{f_{j}(t), g_{j}(t)\right\}_{j=0}^{n-1}$, with respect to $q_{0}$. A set is called admissible if the associated spectral functions, defined in terms of $q_{0}, f_{j}$, and $g_{j}, 0 \leqslant j \leqslant n-1$, satisfy the global relation of Step $(1 \cdot b)$.

Define $q(x, t)$ in terms of these spectral functions by the formula of Step $(1 \cdot a)$. Prove that $q(x, t)$ satisfies the given PDE, and that $q(x, 0)=q_{0}(x),\left(-i \partial_{x}\right)^{j} q(0, t)=f_{j}(t)$, $\left(-i \partial_{x}\right)^{j} q(L, t)=g_{j}(t), 0 \leqslant j \leqslant n-1$.

For equation $(1 \cdot 1)$, the precise definition of admissible functions is given below.

Definition $1 \cdot 3$ (Admissible functions). Let $q_{0}(x) \in \mathbf{C}^{\infty}[0, L]$, and let $\omega(k)$ be defined by equation $(1 \cdot 3)$. Let $\left\{f_{0}(t), \ldots, f_{n-1}(t), g_{0}(t), \ldots, g_{n-1}(t)\right\}$ be a set of $2 n$ sufficiently smooth functions such that $\left(-i \partial_{x}\right)^{j} q_{0}(0)=f_{j}(0),\left(-i \partial_{x}\right)^{j} q_{0}(L)=$ $g_{j}(0), j=0, \ldots, n-1$.

Let

$$
\begin{aligned}
& \hat{F}(k)=\sum_{j=1}^{n} \alpha_{j}\left(\hat{f}_{j-1}(k)+k \hat{f}_{j-2}(k)+\cdots+k^{j-1} \hat{f}_{0}(k)\right), \\
& \hat{G}(k)=\sum_{j=1}^{n} \alpha_{j}\left(\hat{g}_{j-1}(k)+k \hat{g}_{j-2}(k)+\cdots+k^{j-1} \hat{g}_{0}(k)\right),
\end{aligned}
$$


where

$$
\begin{aligned}
& \hat{f}_{j}(k)=\int_{0}^{T} e^{i \omega(k) t} f_{j}(t) d t, \quad j=0, \ldots, n-1, \quad k \in \mathbb{C}, \\
& \hat{g}_{j}(k)=\int_{0}^{T} e^{i \omega(k) t} g_{j}(t) d t, \quad j=0, \ldots, n-1, \quad k \in \mathbb{C} .
\end{aligned}
$$

The set of smooth functions $\left\{f_{0}(t), \ldots, f_{n-1}(t), g_{0}(t), \ldots g_{n-1}(t)\right\}$ is called admissible with respect to $q_{0}(x)$ if and only if the functions $\hat{F}(k)$ and $\hat{G}(k)$ satisfy the following relation:

$$
\hat{F}(k)-e^{-i k L} \hat{G}(k)=-\hat{q}_{0}(k)+e^{i \omega(k) T} \hat{a}(k), \quad k \in \mathbb{C},
$$

where

$$
\hat{a}(k)=\int_{0}^{L} e^{-i k x} a(x) d x,
$$

and $a(x)$ is some function belonging to the space $\mathbf{C}^{\infty}[0, L]$.

For equation (1·1), the implementation of Step 2 yields (see Section 3 ):

Theorem 1.1 (Existence of solutions associated with an admissible set). Assume that $q_{0}(x) \in \mathbf{C}^{\infty}[0, L]$, and that the set of smooth functions $\left\{f_{j}(t), g_{j}(t)\right\}, 0 \leqslant j \leqslant n-1$, is admissible with respect to $q_{0}(x)$, see Definition 1.3.

Let $\hat{q}_{0}(k)$ be defined by equation $(1 \cdot 9)$, and let $\hat{F}(k)$ and $\hat{G}(k)$ be defined by equations $(1 \cdot 13)$ and $(1 \cdot 14)$, respectively.

Define $q(x, t)$ as follows:

$$
\begin{aligned}
q(x, t)= & \frac{1}{2 \pi}\left\{\int_{-\infty}^{\infty} e^{i k x-i \omega(k) t} \hat{q}_{0}(k) d k\right. \\
& \left.+\int_{\partial D_{+}} e^{i k x-i \omega(k) t} \hat{F}(k) d k+\int_{\partial D_{-}} e^{i k(x-L)-i \omega(k) t} \hat{G}(k) d k\right\},
\end{aligned}
$$

where $\omega(k)$ is given by $(1 \cdot 3)$, and $D_{+}$and $D_{-}$are defined by (1.4).

Then:

(i) $q(x, t)$ is the unique solution of equation $(1 \cdot 1)$ such that $t \rightarrow q(\cdot, t)$ is a $\mathbf{C}^{\infty}$ map from $[0, T]$ into $\mathbf{C}^{\infty}[0, L]$;

(ii) $q(x, 0)=q_{0}(x)$;

(iii) $\left(-i \partial_{x}\right)^{j} q(0, t)=f_{j}(t), \quad 0 \leqslant j \leqslant n-1$;

(iv) $\left(-i \partial_{x}\right)^{j} q(L, t)=g_{j}(t), \quad 0 \leqslant j \leqslant n-1$.

Step 3 . Given an appropriate set of boundary conditions at $x=0$ and $x=L$, prove the existence of an admissible set, and compute $\hat{F}(k)$ and $\hat{G}(k)$ in terms of the given initial and boundary conditions.

As a particular concrete example, we prove in Section 4 the existence of an admissible set if the following set of boundary conditions are given:

$$
\left.\begin{array}{ll}
\left(-i \partial_{x}\right)^{j} q(0, t)=u_{j}(t), \quad 0<t<T, \quad 0 \leqslant j \leqslant N-1, \\
\left(-i \partial_{x}\right)^{l} q(L, t)=v_{l}(t), \quad 0<t<T, \quad 0 \leqslant l \leqslant n-N-1,
\end{array}\right\}
$$

with $N$ given by $(1 \cdot 7)$.

In order to state our result, we give some definitions. 
Definition 1.4. The map $\lambda_{l, m}: \overline{D_{R, m}} \rightarrow \overline{D_{R, l}}$ is the biholomorphic map defined by

$$
\omega\left(\lambda_{l, m}(k)\right)=\omega(k), \forall k \in \overline{D_{R, m}} ;
$$

the polynomials $\omega_{n-j}(k), j=0, \ldots, n$, are defined by

$$
\omega_{n-j}(k)=\alpha_{j}+k \alpha_{j+1}+\cdots+\alpha_{n} k^{n-j} .
$$

If $1 \leqslant m \leqslant N$ then $\lambda_{l, m}(k)$ satisfies the following:

$$
1 \leqslant m \leqslant N:\left\{\begin{array}{llc}
\lambda_{l, m}(k) \in D_{+} & \text {if } \quad 1 \leqslant l \leqslant N \\
\lambda_{l, m}(k) \in D_{-} & \text {if } \quad N+1 \leqslant l \leqslant n .
\end{array}\right.
$$

The definition of $\lambda_{l, m}$ implies

$$
\begin{gathered}
\hat{f}_{j}(k)=\hat{f}_{j}\left(\lambda_{l, m}(k)\right), \quad \hat{g}_{j}(k)=\hat{g}_{j}\left(\lambda_{l, m}(k)\right) ; \\
\lambda_{l, m}\left(\lambda_{m, j}(k)\right)=\lambda_{l, j}(k) ; \quad \lambda_{l, m}(k) \sim e^{i(l-m) 2 \pi / n} k, \quad k \rightarrow \infty .
\end{gathered}
$$

In order to state the main theorem, we need one further definition.

Definition $1 \cdot 5$. The $n \times n$ matrix $A$ is defined by

$$
\left(\begin{array}{cccccc}
E_{1} \omega_{n-N-1}\left(\lambda_{1, m}\right) & \ldots & E_{1} \omega_{0}\left(\lambda_{1, m}\right) & -\omega_{N-1}\left(\lambda_{1, m}\right) & \ldots & -\omega_{0}\left(\lambda_{1, m}\right) \\
E_{2} \omega_{n-N-1}\left(\lambda_{2, m}\right) & \ldots & E_{2} \omega_{0}\left(\lambda_{2, m}\right) & -\omega_{N-1}\left(\lambda_{2, m}\right) & \ldots & -\omega_{0}\left(\lambda_{2, m}\right) \\
\cdot & \ldots & \cdot & \cdot & \ldots & \cdot \\
\cdot & \ldots & \cdot & \cdot & \ldots & \cdot \\
E_{N} \omega_{n-N-1}\left(\lambda_{N, m}\right) & \ldots & E_{N} \omega_{0}\left(\lambda_{N, m}\right) & -\omega_{N-1}\left(\lambda_{N, m}\right) & \ldots & -\omega_{0}\left(\lambda_{N, m}\right) \\
\omega_{n-N-1}\left(\lambda_{N+1, m}\right) & \ldots & \omega_{0}\left(\lambda_{N+1, m}\right) & -E_{N+1}^{-1} \omega_{N-1}\left(\lambda_{N+1, m}\right) & \ldots & -E_{N+1}^{-1} \omega_{0}\left(\lambda_{N+1, m}\right) \\
\cdot & \ldots & \cdot & \cdot & \ldots & \cdot \\
\cdot & \ldots & \cdot & \cdot & \ldots & \cdot \\
\omega_{n-N-1}\left(\lambda_{n, m}\right) & \ldots & \omega_{0}\left(\lambda_{n, m}\right) & -E_{n}^{-1} \omega_{N-1}\left(\lambda_{n, m}\right) & \ldots & -E_{n}^{-1} \omega_{0}\left(\lambda_{n, m}\right)
\end{array}\right)
$$

where $E_{l}=\mathrm{e}^{i \lambda_{l, m} L}$, and $\lambda_{l, m}=\lambda_{l, m}(k)$ is the map given by $(1 \cdot 20)$.

Theorem 1.2 (Representation of the solution of the problem (1.1)-(1.2)-(1.19)). Let $q(x, t)$ satisfy equation (1.1), the initial condition $(1 \cdot 2)$ and the boundary conditions (1-19). Assume that $q_{0}(x) \in \mathbf{C}^{\infty}[0, L], u_{j}, v_{l} \in \mathbf{C}^{\infty}[0, T]$ and moreover that $\left(-i \partial_{x}\right)^{j} q_{0}(0)=u_{j}(0), j=0, \ldots, N-1$, and $\left(-i \partial_{x}\right)^{l} q_{0}(L)=v_{l}(0), l=0, \ldots, n-N-1$.

Then $q(x, t)$ is given by equation (1.18), where $\hat{q}_{0}(k)$ is given by $(1 \cdot 9)$, and the spectral functions $\hat{F}(k), \hat{G}(k)$ are defined by the formulas $(1 \cdot 13)$ and $(1 \cdot 14)$, in which $\hat{f}_{j}(k), \hat{g}_{j}(k)$ are constructed as follows:

$$
\left.\begin{array}{l}
\hat{f}_{j}(k)=\hat{u}_{j}(k), \quad 0 \leqslant j \leqslant N-1, \quad k \in \mathbb{C}, \\
\hat{g}_{l}(k)=\hat{v}_{l}(k), \quad 0 \leqslant l \leqslant n-N-1, \quad k \in \mathbb{C},
\end{array}\right\}
$$

where $\hat{u}_{j}(k)$ and $\hat{v}_{l}(k)$ are given by

$$
\hat{u}_{j}(k)=\int_{0}^{T} e^{i \omega(k) t} u_{j}(t) d t, \quad \hat{v}_{l}(k)=\int_{0}^{T} e^{i \omega(k) t} v_{l}(t) d t .
$$

$\hat{f}_{j}(k)$, for $N \leqslant j \leqslant n-1$, and $\hat{g}_{l}(k)$, for $n-N-1 \leqslant l \leqslant n-1$, are given for $k \in D_{R, m}$, 


$$
\begin{aligned}
1 \leqslant m \leqslant N, & \text { by } \\
\hat{f}_{N+j-1}(k)= & \frac{\operatorname{det} B_{j}\left(\lambda_{1, m}(k), \ldots, \lambda_{n, m}(k)\right)-\sum_{h \in \mathbf{Z}} \operatorname{det} B_{j}\left(\lambda_{1, m}\left(k_{h}\right), \ldots, \lambda_{n, m}\left(k_{h}\right)\right)}{\Delta\left(\lambda_{1, m}(k), \ldots, \lambda_{n, m}(k)\right)} \\
& 1 \leqslant j \leqslant n-N, \quad \\
\hat{g}_{j-1}(k)= & \frac{\operatorname{det} B_{j}\left(\lambda_{1, m}(k), \ldots, \lambda_{n, m}(k)\right)-\sum_{h \in \mathbf{Z}} \operatorname{det} B_{j}\left(\lambda_{1, m}\left(k_{h}\right), \ldots, \lambda_{n, m}\left(k_{h}\right)\right)}{\Delta\left(\lambda_{1, m}(k), \ldots, \lambda_{n, m}(k)\right)} \\
& n-N+1 \leqslant j \leqslant n .
\end{aligned}
$$

In the above formulas, $R>0$ is sufficiently large, so that $D_{R,+}$ has $N$ components; the map $\lambda_{l, m}: \overline{D_{R, m}} \rightarrow \overline{D_{R, l}}$ is defined by (1.20); the polynomials $\omega_{n-j}(k)$ are defined by (1.21); $B_{j}\left(\lambda_{1, m}, \ldots, \lambda_{n, m}\right)$ is the matrix obtained by replacing the $j$ th column of the $n \times n$ matrix $A$, defined by (1.23), by the vector $b\left(\lambda_{1, m}, \ldots, \lambda_{n, m}\right)$, whose lth component is given by

$$
b_{l}=\left\{\begin{array}{l}
\sum_{j=1}^{n-N} \omega_{n-j}\left(\lambda_{l, m}\right) \hat{v}_{j-1}(k)-e^{i \lambda_{l, m} L}\left[\sum_{j=1}^{N} \omega_{n-j}\left(\lambda_{l, m}\right) \hat{u}_{j-1}(k)+\hat{q}_{0}\left(\lambda_{l, m}\right)\right], \\
1 \leqslant l \leqslant N \\
e^{-i \lambda_{l, m} L} \sum_{j=1}^{n-N} \omega_{n-j}\left(\lambda_{l, m}\right) \hat{v}_{j-1}(k)-\sum_{j=1}^{N} \omega_{n-j}\left(\lambda_{l, m}\right) \hat{u}_{j-1}(k)+\hat{q}_{0}\left(\lambda_{l, m}\right), \\
\quad N+1 \leqslant l \leqslant n ;
\end{array}\right.
$$

$\Delta$ is the determinant of the $n \times n$ matrix $A$, and $k_{h}$ are the zeros of $\Delta$ in the region $D_{R, m}$.

\section{Remarks}

1. In order to minimize technical difficulties, we have assumed that the given functions $q_{0}(x), u_{j}(t)$ and $v_{l}(t)$ are $\mathbf{C}^{\infty}$ functions. Actually the formulae presented in Proposition 1.2 are valid even if the above functions belong to the functional Sobolev spaces discussed in [7]. The analysis for such spaces is substantially more complicated but it is similar to that presented in [7].

2. The important requirement for well posedness is that $N$ boundary conditions are prescribed at $x=0$ and $n-N$ boundary conditions are prescribed at $x=L$. In this paper, for the sake of concreteness, we have assumed that $\partial_{x}^{j} q(0, t), 0 \leqslant j \leqslant N-1$, and $\partial_{x}^{l} q(L, t), 0 \leqslant l \leqslant n-N-1$, are given. It is straightforward to derive similar formulas for any other $N$ different values of $j$ and $n-N$ different values of $l$. It is also possible to derive similar formulas if linear combinations with constant coefficients of $\partial_{x}^{j} q(0, t)$ and of $\partial_{x}^{l} q(L, t)$ are given (see Example 5·1·2).

3. We recall that the method reviewed in [4] can also be applied to integrable nonlinear PDEs. Indeed, the method presented here, appropriately extended, can be applied to the nonlinear Schrödinger equation in $0<x<L, 0<t<T$. It is interesting that the classical result of the initial value problem of the nonlinear Schrödinger equation with periodic boundary conditions is reproduced as a particular case of this analysis [6].

4. It is straightforward to generalise the formulas presented here to the case of forced equations, i.e. to the case that the right-hand side of equation $(1 \cdot 1)$ is replaced by a given function $F(x, t)$, see [7] for the case of $0<x<\infty$. Thus the results presented here can be useful for the investigation of nonlinear non-integrable PDEs. 
Indeed, in this case the nonlinear terms can be thought of as a forcing and hence at least for short time, or small data, it should be possible to obtain well posedness results.

5. The main difference between the results obtained here and those obtained for $0<x<\infty$ appears in the analysis of the global relation satisfied by the boundary values of $q(x, t)$ and of its derivatives. In our case, this analysis yields a system involving the matrix $A$ which, in contrast to the case of $0<x<\infty$, can be singular. This gives rise to a contribution in the integral representation of $q(x, t)$ which takes the form of sums over the relevant singular points. This can be interpreted as the contribution of the discrete spectrum. The explicit representation of $q(x, t)$ in terms of integrals and of sums is given in Proposition $4 \cdot 1$.

6. It can be shown that the solution representation is independent of $T$. Indeed, all formulae are valid, if $T$ is replaced by $t$ in the definition of $\hat{Q}(k)$ (the proof is analogous to the one given in [7]).

\section{The spectral analysis}

In this section we prove Proposition 1.1.

Equation (1.1) is the compatibility condition of

$$
\left.\begin{array}{l}
\left(\mu(x, t, k) e^{-i k x+i w(k) t}\right)_{x}=e^{-i k x+i w(k) t} q(x, t), \\
\left(\mu(x, t, k) e^{-i k x+i w(k) t}\right)_{t}=-e^{-i k x+i w(k) t} q_{*}(x, t, k),
\end{array}\right\}
$$

where

$$
q_{*}(x, t, k)=\sum_{j=1}^{n} \alpha_{j}\left(\left(-i \partial_{x}\right)^{j-1}+k\left(-i \partial_{x}\right)^{j-2}+\cdots+k^{j-1}\right) q(x, t) .
$$

Equation $2 \cdot 1$ can be written in the form

$$
d\left[e^{-i k x+i \omega(k) t} \mu\right]=w(x, t, k), \quad w(x, t, k)=e^{-i k x+i \omega(k) t}\left[q d x-q_{*} d t\right],
$$

where the differential 1 -form $w$ is closed. Step $(1 \cdot a)$ of the method of [4] is to perform the spectral analysis of equation $(2 \cdot 2)$. This means finding a solution $\mu(x, t, k)$ of equation $(2 \cdot 1)$ bounded in $k \in \mathbb{C}$ for all $(x, t)$ in $[0, L] \times[0, T]$.

Proof of Proposition 1.1. We start by showing that a solution of $(2 \cdot 1)$ bounded in $k \in \mathbb{C}$ has the form

$$
\mu= \begin{cases}\mu_{1}, & k \in E_{+} \\ \mu_{2}, & k \in D_{+} \\ \mu_{3}, & k \in D_{-} \\ \mu_{4}, & k \in E_{-},\end{cases}
$$

where

$$
E=\{k: \operatorname{Im} \omega(k)<0\}, \quad E_{ \pm}=E \cap \mathbb{C}^{ \pm},
$$

and $\mu_{j}(x, t, k)$ are defined below in equation $(2 \cdot 5)$.

Let $z=t+i x$. If $z_{0}$ is an arbitrary constant, then by $\int_{z_{0}}^{z}$ we denote the line integral from $z_{0}$ to $z$. The function

$$
\int_{z_{0}}^{z} e^{i k\left(x-x^{\prime}\right)-i \omega(k)\left(t-t^{\prime}\right)}\left[q\left(x^{\prime}, t^{\prime}\right) d x^{\prime}-q_{*}\left(x^{\prime}, t^{\prime}, k\right) d t^{\prime}\right],
$$


is a particular solution of equation $(2 \cdot 1)$. Moreover, since $w$ is a closed form, this solution does not depend on the choice of the smooth curve between $z_{0}$ and $z$.

It is shown in [5] that there exists a canonical way of choosing $z_{0}$, namely, the corners of the polygon. Thus we define the following 'canonical' particular solutions $[5]:$

$$
\mu_{j}(x, t, k)=\int_{z_{j}}^{z} e^{i k\left(x-x^{\prime}\right)-i \omega(k)\left(t-t^{\prime}\right)}\left[q\left(x^{\prime}, t^{\prime}\right) d x^{\prime}-q_{*}\left(x^{\prime}, t^{\prime}, k\right) d t^{\prime}\right], \quad j=1, \ldots, 4,
$$

where $z_{1}=0, z_{2}=T, z_{3}=T+i L$ and $z_{4}=i L$. The functions $\mu_{j}$ are entire functions of $k$; the general theory presented in [5] implies that these functions are also bounded as $k \rightarrow \infty$ provided that as $k \rightarrow \infty, \mu_{j}$ are defined in the domains indicated in $(2 \cdot 3)$. This also follows from computing the integrals in $(2 \cdot 5)$ along paths parallel to the $x$ and $t$ axis:

$$
\begin{aligned}
& \mu_{1}=\int_{0}^{x} e^{i k\left(x-x^{\prime}\right)} q\left(x^{\prime}, t\right) d x^{\prime}-e^{i k x} \int_{0}^{t} e^{-i \omega(k)\left(t-t^{\prime}\right)} q_{*}\left(0, t^{\prime}\right) d t^{\prime}, \\
& \mu_{2}=\int_{0}^{x} e^{i k\left(x-x^{\prime}\right)} q\left(x^{\prime}, t\right) d x^{\prime}+e^{i k x} \int_{t}^{T} e^{-i \omega(k)\left(t-t^{\prime}\right)} q_{*}\left(0, t^{\prime}\right) d t^{\prime}, \\
& \mu_{3}=-\int_{x}^{L} e^{i k\left(x-x^{\prime}\right)} q\left(x^{\prime}, t\right) d x^{\prime}+e^{i k(x-L)} \int_{t}^{T} e^{-i \omega(k)\left(t-t^{\prime}\right)} q_{*}\left(L, t^{\prime}\right) d t^{\prime}, \\
& \mu_{4}=-\int_{x}^{L} e^{i k\left(x-x^{\prime}\right)} q\left(x^{\prime}, t\right) d x^{\prime}-e^{i k(x-L)} \int_{0}^{t} e^{-i \omega(k)\left(t-t^{\prime}\right)} q_{*}\left(L, t^{\prime}\right) d t^{\prime} .
\end{aligned}
$$

Equation $(2 \cdot 5)$ implies

$$
\left.\begin{array}{l}
\mu_{i}-\mu_{j}=e^{i k x-i \omega(k) t} \rho_{i j}(k), \quad i \neq j . \\
\rho_{i j}(k)=\int_{z_{i}}^{z_{j}} e^{-i k x+i \omega(k) t}\left[q(x, t) d x-q_{*}(x, t, k) d t\right] .
\end{array}\right\}
$$

Computing the integrals in $(2 \cdot 7)$ along paths parallel to the $x$ and $t$ axes, we find

$$
\left.\begin{array}{rlrl}
\mu_{1}-\mu_{4} & =e^{i k x-i \omega(k) t} \hat{q}_{0}(k), & & k \in E_{+} \cap E_{-}, \\
\mu_{2}-\mu_{1}=e^{i k x-i \omega(k) t} \hat{Q}(0, k), & & k \in D_{+} \cap E_{+}, \\
\mu_{2}-\mu_{3}=e^{i k x-i \omega(k) t}\left[\hat{Q}(0, k)-e^{-i k L} \hat{Q}(L, k)+\hat{q}_{0}(k)\right], & & k \in D_{+} \cap D_{-}, \\
\mu_{2}-\mu_{4}=e^{i k x-i \omega(k) t}\left[\hat{q}_{0}(k)+\hat{Q}(0, k)\right], & & k \in D_{+} \cap E_{-}, \\
\mu_{3}-\mu_{4}=e^{i k x-i \omega(k) t} e^{-i k L} \hat{Q}(L, k), & & k \in D_{-} \cap E_{-}, \\
\mu_{3}-\mu_{1}=e^{i k x-i \omega(k) t}\left[-\hat{q}_{0}(k)+e^{-i k L} \hat{Q}(L, k)\right], & & k \in D_{-} \cap E_{+},
\end{array}\right\}
$$

where $\hat{q}_{0}(k), \hat{Q}(0, k) \hat{Q}(L, k)$ are given by equations $(1 \cdot 9)-(1 \cdot 10)$. The expression for $\mu(x, t, k)$ yields the estimate

$$
\mu=O(1 / k), \quad k \rightarrow \infty
$$

In addition, $\mu$ is a sectionally holomorphic function of $k$, as each of its representations $\mu_{j}$ is holomorphic. Equation (2.8) represents the jumps of $\mu$ along the curve separating the domains of analyticity of the various $\mu_{j}$. Thus these equations and the asymptotic estimate $(2 \cdot 9)$ determine a well defined $\mathrm{RH}$ problem for $\mu$; the unique 

solution of this problem is given by [1]

$$
\begin{aligned}
\mu(x, t, k)= & \frac{1}{2 \pi i}\left\{\int_{-\infty}^{\infty} e^{i k^{\prime} x-i \omega\left(k^{\prime}\right) t} \frac{\hat{q}_{0}\left(k^{\prime}\right)}{k^{\prime}-k} d k^{\prime}\right. \\
& \left.+\int_{\partial D_{+}} e^{i k^{\prime} x-i \omega\left(k^{\prime}\right) t} \frac{\hat{Q}\left(0, k^{\prime}\right)}{k^{\prime}-k} d k^{\prime}+\int_{\partial D_{-}} e^{i k^{\prime}(x-L)-i \omega\left(k^{\prime}\right) t} \frac{\hat{Q}\left(L, k^{\prime}\right)}{k^{\prime}-k} d k^{\prime}\right\} .
\end{aligned}
$$

Since $q=\mu_{x}-i k \mu$, differentiating the above expression for $\mu$, with respect to $x$, yields equation $(1 \cdot 8)$.

Since $w$ is a closed form, its integral along the boundary of the polygon vanishes. Writing this relation explicitly for the polygon with vertices $z_{1}, \ldots, z_{4}$, we obtain equation (1.11).

\section{Proof of Theorem 1.1}

We start by making a few technical simplifications, which we summarise in the following lemma. The proof is similar to the one presented in [7].

Lemma 3·1. Given a set of functions $\left\{f_{j}(t), g_{j}(t)\right\}_{j=0}^{n-1}$, admissible with respect to $q_{0}(x)$, let $\hat{f}_{j}(k), \hat{g}_{j}(k)$ be defined as in equations $(1 \cdot 15),(1 \cdot 16)$.

(b) For $k \in D_{R, m}, 1 \leqslant l \leqslant n$, and $1 \leqslant m \leqslant N$,

$$
\sum_{j=1}^{n} \omega_{n-j}\left(\lambda_{l, m}\right) \hat{f}_{j-1}(k)-e^{-i \lambda_{l, m} L} \sum_{j=1}^{n} \omega_{n-j}\left(\lambda_{l, m}\right) \hat{g}_{j-1}(k)=0,
$$

while for $N+1 \leqslant m \leqslant n$,

$$
e^{i \lambda_{l, m} L} \sum_{j=1}^{n} \omega_{n-j}\left(\lambda_{l, m}\right) \hat{f}_{j-1}(k)-\sum_{j=1}^{n} \omega_{n-j}\left(\lambda_{l, m}\right) \hat{g}_{j-1}(k)=0 .
$$

(a) There is no loss of generality in studying the reduced initial boundary value problem obtained when $(1 \cdot 2)$ is replaced by

$$
q(x, 0)=0, \quad x \in[0, L],
$$

and when it is assumed that the admissible functions $f_{j}(t), g_{j}(t), 0 \leqslant j \leqslant n-1$, vanish to all orders at $t=0$.

(c) There is no loss of generality in extending $f_{j}(t), g_{j}(t)$ to belong to the space $\mathbf{C}^{\infty}[0, T+1]$, and to vanish to all orders at $t=T+1$.

(d) If each of the functions $f_{j}(t), g_{j}(t)$ is in $\mathbf{C}^{\infty}[0, T]$, and vanishes to all order at $t=0$ and $t=T$, then the functions $k^{\alpha}\left(d^{\beta} F_{j} / d k^{\beta}\right), k^{\alpha}\left(d^{\beta} G_{j} / d k^{\beta}\right)$ are bounded as $k \rightarrow \infty$ for all $\alpha$, $\beta$ positive integers, $j=0, \ldots, n-1$.

Proof of Theorem 1-1. Given the set of admissible functions $\left\{f_{j}(t), g_{j}(t)\right\}_{j=0}^{n-1}$, define $\hat{F}(k), \hat{G}(k)$ by equations $(1 \cdot 13),(1 \cdot 14)$. Using the polynomials defined by $(1 \cdot 21)$, we can write $\hat{F}(k)$ and $\hat{G}(k)$ as

$$
\hat{F}(k)=\sum_{j=1}^{n} \omega_{n-j}(k) \hat{f}_{j-1}(k), \quad \hat{G}(k)=\sum_{j=1}^{n} \omega_{n-j}(k) \hat{g}_{j-1}(k) .
$$


Define the function $q(x, t)$ as

$$
\begin{aligned}
q(x, t)= & \frac{1}{2 \pi} \sum_{j=1}^{n} \\
& \times\left\{\int_{\partial D_{R,+}} e^{i k x-i \omega(k) t} \omega_{n-j}(k) \hat{f}_{j-1}(k) d k+\int_{\partial D_{R,-}} e^{i k(x-L)-i \omega(k) t} \omega_{n-j}(k) \hat{g}_{j-1}(k) d k\right\} .
\end{aligned}
$$

\section{1. $\mathbf{q}(\mathbf{x}, \mathbf{t})$ solves the equation}

The verification that $q(x, t)$ is in $\mathbf{C}^{\infty}[0, L] \times \mathbf{C}^{\infty}[0, T]$ and that it satisfies equation $(1 \cdot 1)$ follows immediately from the results of Lemma $3 \cdot 1$.

\section{The initial value}

From $(3 \cdot 2)$ we obtain

$$
\begin{aligned}
q(x, 0)= & \frac{1}{2 \pi} \sum_{j=1}^{n} \\
& \times\left\{\int_{\partial D_{R,+}} e^{i k x} \omega_{n-j}(k) \hat{f}_{j-1}(k) d k+\int_{\partial D_{R,-}} e^{i k(x-L)} \omega_{n-j}(k) \hat{g}_{j-1}(k) d k\right\},
\end{aligned}
$$

and since the exponential terms are analytic and bounded in $D_{R,+}$ and $D_{R,-}$ respectively, it follows that $q(x, 0)=0$.

\section{The boundary values}

We show that $\left(-i \partial_{x}\right)^{j} q(0, t)=f_{j}(t), 0 \leqslant j \leqslant n-1$; the verification for the boundary values at $x=L$ is analogous. Evaluating the representation $(3 \cdot 2)$ at $x=0$, we find

$$
\begin{aligned}
q(0, t)= & \frac{1}{2 \pi} \sum_{j=1}^{n} \\
& \times\left\{\int_{\partial D_{R,+}} e^{-i \omega(k) t} \omega_{n-j}(k) \hat{f}_{j-1}(k) d k+\int_{\partial D_{R,-}} e^{-i k L-i \omega(k) t} \omega_{n-j}(k) \hat{g}_{j-1}(k) d k\right\} .
\end{aligned}
$$

Using the property $(a)$ of Lemma $3 \cdot 1$ we can show that

$$
e^{-i k L} \sum_{j=1}^{n} \omega_{n-j}(k) \hat{g}_{j-1}(k)-\sum_{j=1}^{n} \omega_{n-j}(k) \hat{f}_{j-1}(k)=0, \quad k \in D_{R,-} .
$$

Thus equation $(3 \cdot 3)$ becomes

$$
q(0, t)=\frac{1}{2 \pi} \sum_{j=1}^{n} \int_{\partial D_{R}} e^{-i \omega(k) t} \omega_{n-j}(k) \hat{f}_{j-1}(k) d k .
$$

Computing its derivatives with respect to $x$, we obtain

$$
\left(-i \partial_{x}\right)^{l} q(0, t)=\frac{1}{2 \pi} \sum_{j=1}^{n} \int_{\partial D_{R}} e^{-i \omega(k) t} k^{l} \omega_{n-j}(k) \hat{f}_{j-1}(k) d k .
$$


The same argument used in [7] now yields that

$$
\left(-i \partial_{x}\right)^{j} q(0, t)=\frac{1}{2 \pi} \int_{C_{R}} e^{-i t z} \tilde{f}_{j}(z) d z, \quad 0 \leqslant j \leqslant n-1,
$$

where $\tilde{f}_{j}(z)=\int_{0}^{T+1} \mathrm{e}^{i z t} f_{j}(t) d t, C_{r}$ is the contour in the $z$ complex plane, oriented from right to left, given by

$$
C_{r}=\{z \in \mathbb{R},|z|>R\} \cup\{\operatorname{Re} z>0,|z|=R\},
$$

and $k(z)$ is the solution of the equation $z=\omega(k)$ which, for $z \in C_{R}$, lies in $\partial D_{R, 1}$. The Fourier inversion formula yields the claim.

\section{Uniqueness}

Suppose $q_{1}$ and $q_{2}$ are two solutions as in the statement of the theorem. Let $u=$ $q_{1}-q_{2}$. As in [7], it is easy to show that

$$
\frac{d}{d t} \int_{0}^{L}|u(x, t)|^{2} d t \leqslant 0, \quad 0 \leqslant t \leqslant T
$$

Thus $u=0$ and $q_{1}=q_{2}$.

\section{Proof of Theorem $1 \cdot 2$ and an alternative representation}

We now use the definition and properties of an admissible set to show that, if the boundary conditions $(1 \cdot 19)$ are prescribed, then it is possible to compute explicitly the spectral functions $\hat{F}(k)$ and $\hat{G}(k)$. Once these spectral functions are known, the solution $q(x, t)$ can be defined by equation $(1 \cdot 18)$.

Proposition $4 \cdot 1$. Let

$$
\begin{aligned}
& f_{j}(t)=u_{j}(t), \quad 0 \leqslant j \leqslant N-1, \\
& g_{l}(t)=v_{l}(t), \quad 0 \leqslant l \leqslant n-N-1,
\end{aligned}
$$

with $u_{j}(t), v_{l}(t)$ as in $(1 \cdot 19)$.

Then there exist smooth functions $\left\{f_{j}(t)\right\}, j=N, \ldots, n-1$ and $\left\{g_{l}(t)\right\}, l=n-N$, $\ldots, n-1$, such that the set $\left\{f_{j}(t), g_{j}(t)\right\}_{j=0}^{n-1}$ is a set of admissible functions with respect to $q_{0}(x)$. Associated with these admissible functions, there exist the following spectral functions:

$$
\begin{aligned}
& \hat{f}_{j}(k)=\int_{0}^{T} e^{i \omega(k) t} f_{j}(t) d t, \quad j=0, \ldots, n-1, \quad k \in \mathbb{C}, \\
& \hat{g}_{j}(k)=\int_{0}^{T} e^{i \omega(k) t} g_{j}(t) d t, \quad j=0, \ldots, n-1, \quad k \in \mathbb{C} .
\end{aligned}
$$

The solution $q(x, t)$ admits the representation $(1 \cdot 18)$, where the functions $\hat{f}_{j}$ and $\hat{g}_{j}$ are given by equations $(1 \cdot 24)-(1 \cdot 26)$. Alternatively, this representation can be written in 
the form

$$
\begin{aligned}
q(x, t)= & \frac{1}{2 \pi}\left\{\int_{-\infty}^{\infty} e^{i k x-i \omega(k) t} \hat{q}_{0}(k) d k+\int_{\partial D_{R,+}} e^{i k x-i \omega(k) t} \sum_{j=1}^{N} \omega_{n-j}(k) \hat{f}_{j-1}(k) d k\right. \\
& \left.+\int_{\partial D_{R,-}} e^{i k(x-L)-i \omega(k) t} \sum_{j=1}^{n-N} \omega_{n-j}(k) \hat{g}_{j-1}(k) d k\right\} \\
& +\sum_{m=1}^{N} \sum_{j=1}^{n-N}\left[\int_{\partial D_{R, m}} e^{i k x-i \omega(k) t} \frac{\operatorname{det} B_{j}\left(\lambda_{1, m}(k), \ldots, \lambda_{n, m}(k)\right)}{2 \pi \Delta\left(\lambda_{1, m}(k), \ldots, \lambda_{n, m}(k)\right)} d k\right. \\
& \left.+-i \sum_{h} e^{i k_{h} x-i \omega\left(k_{h}\right) t} \frac{\operatorname{det} B_{j}\left(\lambda_{1, m}\left(k_{h}\right), \ldots, \lambda_{n, m}\left(k_{h}\right)\right)}{\Delta^{\prime}\left(\lambda_{1, m}\left(k_{h}\right), \ldots, \lambda_{n, m}\left(k_{h}\right)\right)}\right] \\
& +\sum_{l=N+1}^{n} \sum_{j=n-N+1}^{n}\left[\int_{\partial D_{R, l}} e^{i k(x-L)-i \omega(k) t} \frac{\operatorname{det} B_{j}\left(\lambda_{1, l}(k), \ldots, \lambda_{n, l}(k)\right)}{2 \pi \Delta\left(\lambda_{1, l}(k), \ldots, \lambda_{n, l}(k)\right)} d k\right. \\
& \left.-i \sum_{h} e^{i \tilde{k}_{h}(x-L)-i \omega\left(\tilde{k}_{h}\right) t} \frac{\operatorname{det} B_{j}\left(\lambda_{1, l}\left(\tilde{k}_{h}\right), \ldots, \lambda_{n, l}\left(\tilde{k}_{h}\right)\right)}{\Delta^{\prime}\left(\lambda_{1, l}\left(\tilde{k}_{h}\right), \ldots, \lambda_{n, l}\left(\tilde{k}_{h}\right)\right)}\right],
\end{aligned}
$$

where:

(i) $\hat{f}_{j}, 0 \leqslant j \leqslant N$, and $\hat{g}_{l}, 0 \leqslant l \leqslant n-N$, are given by equation $(1 \cdot 24)$;

(ii) $B_{j}\left(\lambda_{1, m}, \ldots, \lambda_{n, m}\right)$ is the matrix obtained by replacing the $j$ th column of $A\left(\lambda_{1, m}, \ldots, \lambda_{n, m}\right)$ by the vector $b\left(\lambda_{1, m}, \ldots, \lambda_{n, m}\right)$ defined in $(1 \cdot 27)$;

(iii) $\Delta$ is the determinant of the $n \times n$ matrix $A$ defined below by $(1 \cdot 23)$;

(iv) $\left\{k_{h}\right\}$ are the zeros of $\Delta$ in the region $D_{R, m}$;

(v) $\tilde{k}_{h}=\lambda_{l, m}\left(k_{h}\right)$;

(vi) $\Delta^{\prime}$ denotes the derivative of $\Delta$ with respect to $k$.

Proof. We start by observing that, by deforming the contours $\partial D_{-}$and $\partial D_{+}$, equation $(1 \cdot 18)$ can be rewritten in the form

$$
\begin{aligned}
q(x, t)= & \frac{1}{2 \pi}\left\{\int_{-\infty}^{\infty} e^{i k x-i \omega(k) t} \hat{q}_{0}(k) d k\right. \\
& \left.+\sum_{m=1}^{N} \int_{\partial D_{R, m}} e^{i k x-i \omega(k) t} \hat{F}(k) d k+\sum_{l=N+1}^{n} \int_{\partial D_{R, l}} e^{i k(x-L)-i \omega(k) t} \hat{G}(k) d k\right\},
\end{aligned}
$$

with $\hat{F}, \hat{G}$ given by $(1 \cdot 13)$ and $(1 \cdot 14)$, respectively. Thus it is enough to determine the spectral functions $\hat{F}(k), \hat{G}(k)$ for $k \in D_{R}$, where $D_{R}$ is the domain defined in $(1 \cdot 6)$.

Write $\hat{F}(k)$ and $\hat{G}(k)$ in the form $(3 \cdot 1)$. Equation $(1 \cdot 17)$ can be written in the form

$$
e^{i k L} \hat{F}(k)-\hat{G}(k)=-e^{i k L} \hat{q}_{0}(k)+e^{i k L+i \omega(k) T} \hat{a}(k) .
$$

We observe that every term of equation $(1 \cdot 17)$ is bounded in the closure of the domain $D_{-}$, which we denote by $\overline{D_{-}}$; similarly, every term of equation $(4 \cdot 5)$ is bounded if $k \in \overline{D_{+}}$. Let $\lambda_{l, m}(k): D_{R, m} \rightarrow D_{R, l}$ be defined by equation (1.20). Using the definition of the spectral functions $\hat{F}$ and $\hat{G}$, the evaluation of global relation $(4 \cdot 5)$ in $D_{+}$and 
of $(1 \cdot 17)$ in $D_{-}$yields

$$
\left\{\begin{array}{c}
e^{i \lambda_{l, m} L} \sum_{j=1}^{n} w_{n-j}\left(\lambda_{l, m}\right) \hat{f}_{j-1}(k)-\sum_{j=1}^{n} w_{n-j}\left(\lambda_{l, m}\right) \hat{g}_{j-1}(k) \\
=-e^{i \lambda_{l, m} L} \hat{q}_{0}\left(\lambda_{l, m}\right)+e^{i \lambda_{l, m} L+i w(k) T} \hat{a}\left(\lambda_{l, m}\right), \quad 1 \leqslant l \leqslant N \\
\sum_{j=1}^{n} w_{n-j}\left(\lambda_{l, m}\right) \hat{f}_{j-1}(k)-e^{-i \lambda_{l, m} L} \sum_{j=1}^{n} w_{n-j}\left(\lambda_{l, m}\right) \hat{g}_{j-1}(k) \\
=-\hat{q}_{0}\left(\lambda_{l, m}\right)+e^{i w(k) T} \hat{a}\left(\lambda_{l, m}\right), \quad(N+1) \leqslant l \leqslant n
\end{array}\right.
$$

Equation $(4 \cdot 6)$ relates the $2 n$ functions $\hat{f}_{0}(k), \ldots, \hat{f}_{n-1}(k), \hat{g}_{0}(k), \ldots, \hat{g}_{n-1}(k)$; however, they also contain the arbitrary function $\hat{a}(k)$.

Moving to the right-hand side the terms containing the known data $\hat{f}_{j-1}(k), j=$ $1, \ldots, N$, and $\hat{g}_{j-1}(k), j=1, \ldots, n-N$, we obtain the following system for the spectral functions $\hat{f}_{j}, j=N, \ldots, n-1$ and $\hat{g}_{l}(k), l=N-n, \ldots, n-1$ :

$$
\left\{\begin{array}{l}
e^{i \lambda_{l, m} L} \sum_{j=N+1}^{n} w_{n-j}\left(\lambda_{l, m}\right) \hat{f}_{j-1}(k)-\sum_{j=n-N+1}^{n} w_{n-j}\left(\lambda_{l, m}\right) \hat{g}_{j-1}(k) \\
\quad=b_{l}+e^{i \lambda_{l, m} L+i w(k) T} \hat{a}\left(\lambda_{l, m}\right), \quad 1 \leqslant l \leqslant N, \\
\sum_{j=n-N+1}^{n} w_{n-j}\left(\lambda_{l, m}\right) \hat{f}_{j-1}(k)-e^{-i \lambda_{l, m} L} \sum_{j=N+1}^{n} w_{n-j}\left(\lambda_{l, m}\right) \hat{g}_{j-1}(k) \\
\quad=b_{l}+e^{i w(k) T} \hat{a}\left(\lambda_{l, m}\right), \quad(N+1) \leqslant l \leqslant n,
\end{array}\right.
$$

where $\left\{b_{l}\right\}_{l=1}^{n}$ is given by equation (1.27). It is important to note that all terms in this system are holomorphic functions, bounded for $k \in D_{R, m}, 1 \leqslant m \leqslant N$.

We now write the system $(4 \cdot 7)$ in matrix form as

$$
\begin{aligned}
A\left(\lambda_{1, m}, \ldots, \lambda_{n, m}\right)\left(\hat{f}_{N}(k), \ldots, \hat{f}_{n-1}(k), \hat{g}_{n-N}(k), \ldots, \hat{g}_{n-1}(k)\right)^{\tau} \\
=b\left(\lambda_{1, m}, \ldots, \lambda_{n, m}\right)+\mathrm{e}^{i \omega(k) T} c\left(\lambda_{1, m}, \ldots, \lambda_{n, m}\right) .
\end{aligned}
$$

Here:

(i) $A$ is the $n \times n$ matrix defined by (1.23);

(ii) $b=\left(b_{1}, \ldots, b_{N}, b_{N+1}, \ldots, b_{n}\right)^{\tau}$ is the $n$-vector whose components are defined in $(1 \cdot 27)$;

(iii) $c\left(\lambda_{1, m}, \ldots, \lambda_{n, m}\right)$ is an $n$-vector whose $l$ th component is given by

$$
c_{l}= \begin{cases}e^{i \lambda_{l, m} L+i \omega(k) T} \hat{a}\left(\lambda_{l, m}\right), & 1 \leqslant l \leqslant N, \\ e^{i \omega(k) T} \hat{a}\left(\lambda_{l, m}\right), & N+1 \leqslant l \leqslant n ;\end{cases}
$$

(iv) $(\cdot)^{\tau}$ denotes transposition.

Let $\Delta=\operatorname{det}(A)$. By induction, one can show that this determinant is always of the form

$$
p\left(E_{1}, E_{2}, \ldots, E_{N}, E_{N+1}^{-1}, \ldots, E_{n}^{-1}\right)+r(k),
$$

where $p$ is a polynomial in the exponentials $E_{1}, \ldots, E_{N}, E_{N+1}^{-1}, E_{n}^{-1}$ with coefficients depending on $k$, and $r(k)$ is a polynomial function of $k$ of degree at most $n(n+1) / 2$, with zero-th order term $r_{0} \neq 0$. Since the exponentials $E_{1}, \ldots, E_{N}, E_{N+1}^{-1}, E_{n}^{-1}$ are analytic, bounded and decay as $k \rightarrow \infty$, for $k \in D_{R, m}, 1 \leqslant m \leqslant N$, the term $1 / \Delta$ is bounded for $k \in D_{R, m}$, except possibly for poles. We can use Cramer's rule, to solve this system explicitly for the functions $\hat{f}_{N}(k), \ldots, \hat{f}_{n-1}(k), \hat{g}_{n-N}(k), \ldots, \hat{g}_{n-1}(k)$, for 
$k \in D_{R, m}:$

$$
\begin{aligned}
\hat{f}_{N+j-1}(k)= & \frac{\operatorname{det} B_{j}\left(\lambda_{1, m}, \ldots, \lambda_{n, m}\right)}{\Delta\left(\lambda_{1, m}, \ldots, \lambda_{n, m}\right)}+e^{i \omega(k) T} \frac{\operatorname{det} C_{j}\left(\lambda_{1, m}, \ldots, \lambda_{n, m}\right)}{\Delta\left(\lambda_{1, m}, \ldots, \lambda_{n, m}\right)}, \\
& 1 \leqslant j \leqslant n-N, \\
\hat{g}_{j-1}(k)= & \frac{\operatorname{det} B_{j}\left(\lambda_{1, m}, \ldots, \lambda_{n, m}\right)}{\Delta\left(\lambda_{1, m}, \ldots, \lambda_{n, m}\right)}+e^{i \omega(k) T} \frac{\operatorname{det} C_{j}\left(\lambda_{1, m}, \ldots, \lambda_{n, m}\right)}{\Delta\left(\lambda_{1, m}, \ldots, \lambda_{n, m}\right)}, \\
& n-N+1 \leqslant j \leqslant n,
\end{aligned}
$$

where $B_{j}\left(\lambda_{1, m}, \ldots, \lambda_{n, m}\right)$ is the matrix obtained by replacing the $j$ th column of $A\left(\lambda_{1, m}, \ldots, \lambda_{n, m}\right)$ by the vector $b\left(\lambda_{1, m}, \ldots, \lambda_{n, m}\right)$, and $C_{j}\left(\lambda_{1, m}, \ldots, \lambda_{n, m}\right)$ is the matrix obtained by replacing the $j$ th column of $A\left(\lambda_{1, m}, \ldots, \lambda_{n, m}\right)$ by the vector $c\left(\lambda_{1, m}, \ldots, \lambda_{n, m}\right)$.

The spectral functions $\hat{f}_{N+j-1}(k)$ and $\hat{g}_{j-1}(k)$ on the left-hand side of equations $(4 \cdot 9)-(4 \cdot 10)$ are holomorphic, bounded functions in $D_{R, m}$; moreover, since they are the Fourier transform of $\mathbf{C}^{\infty}$ functions, they must have at least $O\left(|k|^{-j}\right)$ decay as $k \rightarrow \infty$. Equations (4.9) and (4.10) define these functions as the unique solution of the well defined system (4.7); thus the right-hand side of both equations (4.9) and $(4 \cdot 10)$ must have the same analyticity and boundedness properties. However the determinant $\Delta(k)$ may have zeroes in $D_{R, m}, 1 \leqslant m \leqslant N$. For equations (4.9)(4.10) to be meaningful, we must subtract the contribution of the associated poles. Namely, suppose that $\Delta\left(\lambda_{1, m}\left(k_{0}\right), \ldots, \lambda_{n, m}\left(k_{0}\right)\right)=0, k_{0} \in D_{R, m}$. Since $\Delta \hat{f}_{N+j-1}=$ $\operatorname{det} B_{j}+e^{i \omega(k) T} \operatorname{det} C_{j}$, and similarly for $\hat{g}_{j-1}$, it must be that, for $1 \leqslant j \leqslant n$,

$$
\operatorname{det} B_{j}\left(\lambda_{1, m}\left(k_{0}\right), \ldots, \lambda_{n, m}\left(k_{0}\right)\right)+e^{i \omega\left(k_{0}\right) T} \operatorname{det} C_{j}\left(\lambda_{1, m}\left(k_{0}\right), \ldots, \lambda_{n, m}\left(k_{0}\right)\right)=0 .
$$

As the function $\hat{a}(k)$ in the definition of the matrix $C$ is unspecified, we can choose it in such a way that the latter equation is satisfied. Hence, we can subtract the resulting zero term, divided by $\Delta$, from the expressions $(4 \cdot 9)$ and $(4 \cdot 10)$, and obtain

$$
\begin{aligned}
\hat{f}_{N+j-1}(k)= & \frac{\operatorname{det} B_{j}\left(\lambda_{1, m}(k), \ldots, \lambda_{n, m}(k)\right)-\operatorname{det} B_{j}\left(\lambda_{1, m}\left(k_{0}\right), \ldots, \lambda_{n, m}\left(k_{0}\right)\right)}{\Delta\left(\lambda_{1, m}(k), \ldots, \lambda_{n, m}(k)\right)} \\
& +e^{i \omega(k) T} \frac{\operatorname{det} C_{j}\left(\lambda_{1, m}(k), \ldots, \lambda_{n, m}(k)\right)-\operatorname{det} C_{j}\left(\lambda_{1, m}\left(k_{0}\right), \ldots, \lambda_{n, m}\left(k_{0}\right)\right)}{\Delta\left(\lambda_{1, m}(k), \ldots, \lambda_{n, m}(k)\right)} \\
1 \leqslant j \leqslant n-N, & \\
\hat{g}_{j-1}(k)= & \frac{\operatorname{det} B_{j}\left(\lambda_{1, m}(k), \ldots, \lambda_{n, m}(k)\right)-\operatorname{det} B_{j}\left(\lambda_{1, m}\left(k_{0}\right), \ldots, \lambda_{n, m}\left(k_{0}\right)\right)}{\Delta\left(\lambda_{1, m}(k), \ldots, \lambda_{n, m}(k)\right)} \\
& +e^{i \omega(k) T} \frac{\operatorname{det} C_{j}\left(\lambda_{1, m}(k), \ldots, \lambda_{n, m}(k)\right)-\operatorname{det} C_{j}\left(\lambda_{1, m}\left(k_{0}\right), \ldots, \lambda_{n, m}\left(k_{0}\right)\right)}{\Delta\left(\lambda_{1, m}(k), \ldots, \lambda_{n, m}(k)\right)} \\
& n-N+1 \leqslant j \leqslant n .
\end{aligned}
$$

The terms appearing on the right-hand side of equations $(4 \cdot 11)-(4 \cdot 12)$ are nonsingular at $k=k_{0}$, and define holomorphic functions in $D_{R, m}, 1 \leqslant m \leqslant N$; moreover, because of the presence of the nonzero polynomial term $r(k)$ in the expression of the determinant $\Delta(k)$, all these terms are also bounded and decaying. Hence equations $(4 \cdot 11)-(4 \cdot 12)$ are compatible with the known properties of the spectral functions. 
We now show that the terms containing $\hat{a}(k)$ do not contribute to the representation of $q(x, t)$. Indeed, the exponential term $e^{i k x+i \omega(k)(T-t)}$ is bounded and analytic for $k \in D_{R,+}, 1 \leqslant m \leqslant N$; thus it follows that the integral

$$
\int_{\partial D_{R, m}} e^{i k x-i \omega(k) t} \omega_{n-j}(k) e^{i \omega(k) T} \frac{\operatorname{det} C\left(\lambda_{1, m}, \ldots, \lambda_{n, m}\right)}{\Delta\left(\lambda_{1, m}, \ldots, \lambda_{n, m}\right)} d k
$$

vanishes, except if the function $\Delta$ has zeroes inside the domain $D_{R,+}$, and the integration of the terms containing the exponential $e^{i k x-i \omega(k)(t-T)}$ is zero. Thus the term containing the function $\hat{a}(k)$ can be dropped from the system $(4 \cdot 7)$, and we can write the representation of the solution in the form (1-18).

We also note that computing explicitly in the integral representation $(1 \cdot 18)$ the contribution of the poles of $1 / \Delta$, we obtain the explicit representation (4.3). Namely, consider the integral along $\partial D_{R, m}, 1 \leqslant m \leqslant N$, of the function $\hat{f}_{N+j-1}(k)$ multiplied by the exponential $e^{i k x+i \omega(k)(T-t)}$; for $1 \leqslant j \leqslant n-N$, we obtain

$$
\begin{aligned}
\int_{D_{R,+}} e^{i k x-i \omega(k) t} \hat{f}_{N+j-1}(k) d k= & \sum_{m=1}^{N}\left[\int_{D_{R, m}} e^{i k x-i \omega(k) t} \frac{\operatorname{det} B_{j}\left(\lambda_{1, m}(k), \ldots, \lambda_{n, m}(k)\right)}{\Delta\left(\lambda_{1, m}(k), \ldots, \lambda_{n, m}(k)\right)} d k .\right. \\
& \left.-2 \pi i e^{i k_{0} x-i \omega\left(k_{0}\right) t} \frac{\operatorname{det} B_{j}\left(\lambda_{1, m}\left(k_{0}\right), \ldots, \lambda_{n, m}\left(k_{0}\right)\right)}{\Delta^{\prime}\left(\lambda_{1, m}\left(k_{0}\right), \ldots, \lambda_{n, m}\left(k_{0}\right)\right)}\right]
\end{aligned}
$$

Similarly, for $n-N+1 \leqslant j \leqslant n$, if $k_{0}$ is a zero of $\Delta$ in $D_{R, l}$,

$$
\begin{aligned}
\int_{D_{R,-}} e^{i k(x-L)-i \omega(k) t} \hat{g}_{j-1}(k) d k & =\sum_{l=N+1}^{n}\left[\int_{D_{R, l}} e^{i k(x-L)-i \omega(k) t} \frac{\operatorname{det} B_{j}\left(\lambda_{1, l}(k), \ldots, \lambda_{n, l}(k)\right)}{\Delta\left(\lambda_{1, l}(k), \ldots, \lambda_{n, l}(k)\right)} d k\right. \\
& \left.-2 \pi i e^{i k_{0}(x-L)-i \omega\left(k_{0}\right) t} \frac{\operatorname{det} B_{j}\left(\lambda_{1, l}\left(k_{0}\right), \ldots, \lambda_{n, l}\left(k_{0}\right)\right)}{\Delta^{\prime}\left(\lambda_{1, l}\left(k_{0}\right), \ldots, \lambda_{n, l}\left(k_{0}\right)\right)}\right] .
\end{aligned}
$$

The generalization of this case to any finite number of poles in straightforward. This representation is valid also in the case of infinitely many poles, provided that the relevant sums converge.

Remark $4 \cdot 1$. It is important to stress that the proof given above depends crucially on the fact that the term $1 / \operatorname{det}(A)$ is bounded for $k \in D_{R, m}$. This is not the case for all sets of $n$ given boundary conditions (see Remark $5 \cdot 2 \cdot 2$ below for an example).

\section{Examples}

We compute explicitly the representation of the solution of some illustrative boundary value problems for a second and a third order equation.

Example 5·1.

$$
i q_{t}+q_{x x}=0, \quad 0<x<L, \quad 0<t<T .
$$

The closed 1-form associated to this equation is given by

$$
w(x, t, k)=e^{-i k x+i k^{2} t}\left[q d x+\left(i q_{x}+k q\right) d t\right]
$$




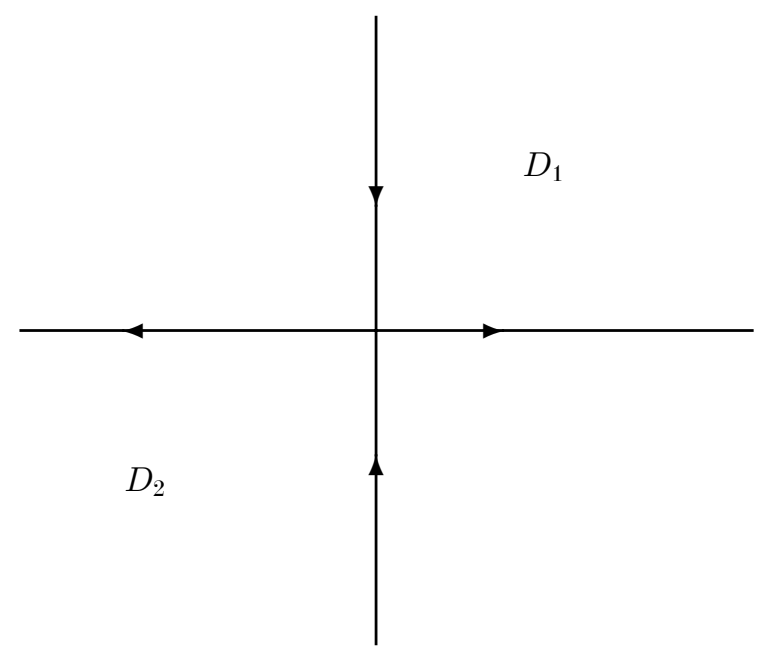

Fig. 1. The domains $D_{+}=D_{1}$ and $D_{-}=D_{2}$ for the equation $i q_{t}+q_{x x}=0$.

Thus $\omega(k)=k^{2}$, and $D_{+}=D_{1}, D_{-}=D_{2}$ are the domains shown in Fig. 1 and defined by

$$
D_{1}=\left\{k: 0 \leqslant \arg (k) \leqslant \frac{\pi}{2}\right\}, \quad D_{2}=\left\{k: \pi \leqslant \arg (k) \leqslant \frac{3 \pi}{2}\right\} .
$$

The biholomorphic maps $\lambda_{l, m}(k)$ are given by

$$
\lambda_{1,1}(k)=k, \quad \lambda_{2,1}(k)=-k .
$$

A set of admissible functions with respect with the given initial data $q_{0}(x)=q(x, 0)$ contains four functions, $f_{0}(t), f_{1}(t), g_{0}(t)$, and $g_{1}(t)$. The spectral functions are given by

$$
\begin{aligned}
& \hat{F}(k)=\int_{0}^{T} e^{i k^{2} t}\left[f_{1}(t)+k f_{0}(t)\right] d t=\hat{f}_{1}(k)+k \hat{f}_{0}(k), \\
& \hat{G}(k)=\int_{0}^{T} e^{i k^{2} t}\left[g_{1}(t)+k g_{0}(t)\right] d t=\hat{g}_{1}(k)+k \hat{g}_{0}(k) .
\end{aligned}
$$

Hence, for $k \in D_{1}$, the global relation yields

$$
\left.\begin{array}{l}
e^{i k L}\left[\hat{f}_{1}(k)+k \hat{f}_{0}(k)\right]-\left[\hat{g}_{1}(k)+k \hat{g}_{0}(k)\right]=-e^{i k L} \hat{q}_{0}(k)+e^{i k^{2} T+i k L} \hat{a}(k), \\
{\left[\hat{f}_{1}(k)-k \hat{f}_{0}(k)\right]-e^{i k L}\left[\hat{g}_{1}(k)-k \hat{g}_{0}(k)\right]=-\hat{q}_{0}(-k)+e^{i k^{2} T} \hat{a}(-k) .}
\end{array}\right\}
$$

These are two equations for the four functions $\hat{f}_{0}(k), \hat{g}_{0}(k), \hat{f}_{1}(k)$ and $\hat{g}_{1}(k)$. By assigning appropriately two boundary conditions, these equations yield a system of two equations for two unknowns, admitting a unique solution.

Although we have only shown this for the particular case that the value of the equation is given as boundary condition both at $x=0$ and $x=L$, the same holds for other sets of boundary conditions (see Example $5 \cdot 1 \cdot 2$ below). 
5.1.1 The boundary value problem for equation (5.1) defined by the initial and boundary conditions

$$
\begin{aligned}
q(x, 0) & =q_{0}(x), & & 0<x<L, \\
q(0, t) & =u_{0}(t), & & q(L, t)=v_{0}(t) \quad 0<t<T,
\end{aligned}
$$

where $q_{0}(x), u_{0}(t)$ and $v_{0}(t)$ are smooth functions, compatible at $x=t=0$ and at $x=L, t=0$, is well posed. Its unique solution is given by

$$
\begin{aligned}
q(x, t)= & \frac{1}{2 \pi}\left\{\int_{-\infty}^{\infty} e^{i k x-i k^{2} t} \hat{q}_{0}(k) d k+\int_{\partial D_{1}} e^{i k x-i k^{2} t} k \hat{u}_{0}(k) d k+\int_{\partial D_{2}} e^{i k(x-L)-i k^{2} t} k \hat{v}_{0}(k) d k\right\} \\
& +\left[\int_{\partial D_{1}} e^{i k x-i k^{2} t} \frac{b_{2}(k)-e^{i k L} b_{1}(k)}{2 \pi\left(1-e^{2 i k L}\right)} d k+\sum_{h \in \mathbb{Z}} e^{i k_{h} x-i k_{h}^{2} t} \frac{b_{2}\left(k_{h}\right)-e^{i k_{h} L} b_{1}\left(k_{h}\right)}{2 L e^{2 i k L}}\right] \\
& +\left[\int_{\partial D_{2}} e^{i k(x-L)-i k^{2} t} \frac{e^{-i k L} b_{2}(-k)-b_{1}(-k)}{2 \pi\left(1-e^{-2 i k L}\right)} d k\right. \\
& \left.+\sum_{h \in \mathbb{Z}} e^{-i k_{h}(x-L)-i k_{h}^{2} t} \frac{e^{i k_{h} L} b_{2}\left(k_{h}\right)-b_{1}\left(k_{h}\right)}{2 L e^{2 i k_{h} L}}\right],
\end{aligned}
$$

where $\hat{q}_{0}(k)$ is defined by $(1 \cdot 9)$, the functions $\hat{u}_{0}(k), \hat{v}_{0}(k), b_{1}(k), b_{2}(k)$ are given by

$$
\begin{gathered}
\hat{u}_{0}(k)=\int_{0}^{T} e^{i k^{2} t} u_{0}(t) d t, \quad \hat{v}_{0}(k)=\int_{0}^{T} e^{i k^{2} t} v_{0}(t) d t, \\
b_{1}=-e^{i k L} \hat{q}_{0}(k)-k e^{i k L} \hat{u}_{0}(k)+k \hat{v}_{0}(k), \quad b_{2}=-\hat{q}_{0}(-k)+k \hat{u}_{0}(k)-e^{i k L} k \hat{v}_{0}(k),
\end{gathered}
$$

and $k_{h}$ are given by

$$
k_{h}=h \pi / L, \quad h \in \mathbb{Z} .
$$

Indeed, let $E=e^{i k L}$. The known functions $\hat{f}_{0}(k)$ and $\hat{g}_{0}(k)$, are given by

$$
\hat{f}_{0}(k)=\hat{u}_{0}(k), \quad \hat{g}_{0}(k)=\hat{v}_{0}(k) .
$$

For $k \in D_{1}$, the system $(5 \cdot 2)$ can be used to define $\hat{f}_{1}(k)$ and $\hat{g}_{1}(k)$

$$
\left(\begin{array}{cc}
E & -1 \\
1 & -E
\end{array}\right)\left(\begin{array}{c}
\hat{f}_{1}(k) \\
\hat{g}_{1}(k)
\end{array}\right)=\left(\begin{array}{c}
b_{1} \\
b_{2}
\end{array}\right)+\left(\begin{array}{l}
a_{1} \\
a_{2}
\end{array}\right)
$$

where $b_{1}(k), b_{2}(k)$ are given by $(5 \cdot 7)$, and

$$
a_{1}=E e^{i k^{2} T} \hat{a}(k), \quad a_{2}=e^{i k^{2} T} \hat{a}(-k) .
$$

The determinant $\Delta$ of this system is given by

$$
\Delta(k)=1-E^{2}=1-e^{2 i k L},
$$

and its solution is given by

$$
\begin{aligned}
\hat{f}_{1}(k) & =\frac{b_{2}+a_{2}-E\left(b_{1}+a_{1}\right)}{\Delta} \\
\hat{g}_{1}(k) & =\frac{E\left(b_{2}+a_{2}\right)-\left(b_{1}+a_{1}\right)}{\Delta}, \quad k \in D_{1} .
\end{aligned}
$$

The terms containing $a_{1}$ and $a_{2}$ can be used to subtract the contribution of the poles arising from the zeros $k_{h}$ of $1-E^{2}$, given by $(5 \cdot 8)$. Indeed, choose $a_{1}$ and $a_{2}$ such 
that $a_{i}\left(k_{h}\right)=-b_{i}\left(k_{h}\right), i=1,2$; then $\hat{f}_{1}$ and $\hat{g}_{1}$ can be written as

$$
\left.\begin{array}{l}
\hat{f}_{1}(k)=\frac{b_{2}+a_{2}-E\left(b_{1}+a_{1}\right)-\left[b_{2}+a_{2}-E\left(b_{1}+a_{1}\right)\right]\left(k_{h}\right)}{\Delta} \\
\hat{g}_{1}(k)=\frac{E\left(b_{2}+a_{2}\right)-\left(b_{1}+a_{1}\right)-\left[E\left(b_{2}+a_{2}\right)-\left(b_{1}+a_{1}\right)\right]\left(k_{h}\right)}{\Delta}, \quad k \in D_{1} .
\end{array}\right\}
$$

The expressions (5.11) now represent two bounded holomorphic functions.

Multiplying $\hat{f}_{1}(k)$ by $e^{i k x-i k^{2} t}$, and integrating along $\partial D_{1}$, and similarly, multiplying $\hat{g}_{1}(k)$ by $e^{i k(L-x)-i k^{2} t}$, and integrating along $\partial D_{2}$, it is easy to show that the terms containing $a_{1}$ and $a_{2}$ give no contribution, as the overall exponential is bounded in the whole of the region. We can also compute explicitly the residues due to the poles in the remaining terms; this gives

$$
i \sum_{h=-\infty}^{\infty} e^{i k_{h} x-i k_{n}^{2} t} \frac{b_{2}\left(k_{h}\right)-E\left(k_{h}\right) b_{1}\left(k_{h}\right)}{\Delta^{\prime}\left(k_{h}\right)}, \quad i \sum_{h=-\infty}^{\infty} e^{-i k_{h}(x-L)-i k_{n}^{2} t} \frac{b_{1}\left(k_{h}\right)-E\left(k_{h}\right) b_{2}\left(k_{h}\right)}{\Delta^{\prime}\left(k_{h}\right)} .
$$

Replacing the above functions in the representation $(4 \cdot 3)$, we obtain $(5 \cdot 5)$. Note that the two sums in $(5 \cdot 12)$ can be rewritten as

$$
-\frac{1}{2 L}\left[\sum_{h=-\infty}^{\infty} e^{i \pi h x / L-i(\pi h / L)^{2} t} b_{2}\left(\frac{h \pi}{L}\right)-\sum_{h=-\infty}^{\infty} e^{i \pi h x / L-i(\pi h / L)^{2} t}(-1)^{h} b_{1}\left(\frac{h \pi}{L}\right)\right],
$$

and

$$
\frac{1}{2 L}\left[\sum_{h=-\infty}^{\infty} e^{-i \pi h(x-L) / L-i(\pi h / L)^{2} t} b_{1}\left(\frac{h \pi}{L}\right)-\sum_{h=-\infty}^{\infty} e^{-i \pi h(x-L) / L-i(\pi h / L)^{2} t}(-1)^{h} b_{2}\left(\frac{h \pi}{L}\right)\right] .
$$

Since the functions $b_{1}(k), b_{2}(k)$ depend only on the Fourier coefficients $\hat{q}_{0}(k), \hat{u}_{0}\left(k^{2}\right)$, $\hat{v}_{0}\left(k^{2}\right)$, if the given data $q_{0}(x), u_{0}(t), u_{0}(t)$ are sufficiently smooth both the trigonometric series in $(5 \cdot 12)$ converge.

5.1.2 The boundary value problem for equation (5.1) defined by the initial condition $(5 \cdot 3)$ and by the boundary condition

$$
\left.\begin{array}{l}
q_{x}(0, t)+\beta q(0, t)=u_{0}(t), \\
q_{x}(L, t)+\beta q(L, t)=v_{0}(t), \quad 0<t<T,
\end{array}\right\}
$$

where $\beta$ is a constant, is well posed. The solution, for the case that $u_{0}=v_{0}=0$, is given by

$$
\begin{aligned}
q(x, t)= & \frac{1}{2 \pi} \int_{-\infty}^{\infty} e^{i k x-i k^{2} t} \hat{q}_{0}(k) d k+\frac{2 \beta e^{i \beta^{2} t}}{e^{-2 \beta L}-1}\left[e^{-\beta x} \hat{q}_{0}(-i \beta)-e^{\beta x+2 \beta L} \hat{q}_{0}(i \beta)\right] \\
& +\sum_{h \in \mathbb{Z}} e^{i k_{h} x-i k_{h}^{2} t} \frac{\left[e^{2 i k_{h} L} \hat{q}_{0}\left(k_{h}\right)-\left(i \beta+k_{h} / i \beta-k_{h}\right) \hat{q}_{0}\left(-k_{h}\right)\right]}{2 L e^{2 i k_{h} L}} \\
& -\sum_{h \in \mathbb{Z}} e^{-i k_{h}(x-2 L)-i k_{h}^{2} t} \frac{\left[\hat{q}_{0}\left(-k_{h}\right)-\left(i \beta-k_{h} / i \beta+k_{h}\right) \hat{q}_{0}\left(k_{h}\right)\right]}{2 L e^{2 i k_{h} L}}
\end{aligned}
$$

where $\hat{q}_{0}(k)$ is defined by $(1 \cdot 9)$ and $k_{h}$ are given by (5.8). A similar expression is found when $u, v \neq 0$. 
Let $E$ be defined as in $5 \cdot 1 \cdot 1$. The boundary condition implies that

$$
\hat{f}_{1}(k)=i \beta \hat{f}_{0}(k)+\hat{u}_{0}(k), \quad \hat{g}_{1}(k)=i \beta \hat{g}_{0}(k)+\hat{v}_{0}(k) ;
$$

thus the system $(5 \cdot 2)$ becomes

$$
\left(\begin{array}{cc}
E(i \beta+k) & -(i \beta+k) \\
(i \beta-k) & -E(i \beta-k)
\end{array}\right)\left(\begin{array}{c}
\hat{f}_{0}(k) \\
\hat{g}_{0}(k)
\end{array}\right)=\left(\begin{array}{c}
b_{1} \\
b_{2}
\end{array}\right)+\left(\begin{array}{c}
a_{1} \\
a_{2}
\end{array}\right),
$$

where

$$
b_{1}(k)=\hat{v}_{0}(k)-E \hat{u}_{0}(k)-E \hat{q}_{0}(k), \quad b_{2}(k)=E \hat{v}_{0}(-k)-\hat{u}_{0}(-k)-\hat{q}_{0}(-k),
$$

$a_{1}, a_{2}$ are given by equation $(5 \cdot 9)$, and $\hat{q}_{0}(k)$ is given by $(1 \cdot 9)$. The determinant of this system is given by

$$
\Delta=\left(E^{2}(k)-1\right)\left(\beta^{2}+k^{2}\right) .
$$

Since $\lim _{k \rightarrow \infty} 1 / \Delta=0$, the system can be solved uniquely; its solution is given by

$$
\begin{aligned}
& \hat{f}_{0}(k)=\frac{1}{\Delta}\left[(i \beta+k)\left(b_{2}+a_{2}\right)-E(i \beta-k)\left(b_{1}+a_{1}\right)\right], \\
& \hat{g}_{0}(k)=\frac{1}{\Delta}\left[E(i \beta+k)\left(b_{2}+a_{2}\right)-(i \beta-k)\left(b_{1}+a_{1}\right)\right], \quad k \in D_{1} .
\end{aligned}
$$

Substituting these spectral functions in the general representation (1-18), and dropping the terms containing $a_{1}$ or $a_{2}$, we find that the integrand of the integral along $\partial D_{1}$ is

$$
\begin{aligned}
\frac{e^{i k x-i k^{2} t}}{1-E^{2}(k)}\left[E^{2}(k)\left(\hat{q}_{0}(k)-\hat{u}_{0}(k)\right)-E \hat{v}_{0}(k)\right. & \\
& \left.+\frac{i \beta+k}{i \beta-k}\left(E \hat{v}_{0}(-k)-\hat{u}_{0}(-k)-\hat{q}_{0}(-k)\right)\right]+e^{i k x-i k^{2} t} \hat{u}_{0}(k),
\end{aligned}
$$

while the integrand of the integral along $\partial D_{2}$ is

$$
\begin{aligned}
& \frac{e^{i k(x-L)-i k^{2} t}}{E^{2}(-k)-1}\left[E(-k)\left(\hat{q}_{0}(k)-\hat{u}_{0}(-k)\right)-\hat{v}_{0}(-k)\right. \\
&\left.+\frac{i \beta+k}{i \beta-k}\left(E(-k) \hat{v}_{0}(-k)-\hat{u}_{0}(-k)-\hat{q}_{0}(-k)\right)\right]+e^{i k(x-L)-i k^{2} t} \hat{v}_{0}(-k) .
\end{aligned}
$$

The zeros of the functions $E^{2}-1$ and $i \alpha-k$ inside $D_{1}$ occur at the points $k=k_{h}$, with $k_{h}$ given by $(5 \cdot 8)$, and at the point $k=i \alpha$. Computing the contribution due to these zeros, and simplifying the result when $u_{0}=v_{0}=0$, we obtain the representation $(5 \cdot 14)$.

\section{Example 5·2.}

$$
q_{t}+q_{x x x}=0, \quad 0<x<L, \quad 0<t<T .
$$

The closed 1-form associated to this equation is given by

$$
w(x, t, k)=e^{-i k x-i k^{3} t}\left[q d x-\left(q_{x x}+i k q_{x}-k^{2} q\right) d t\right] .
$$




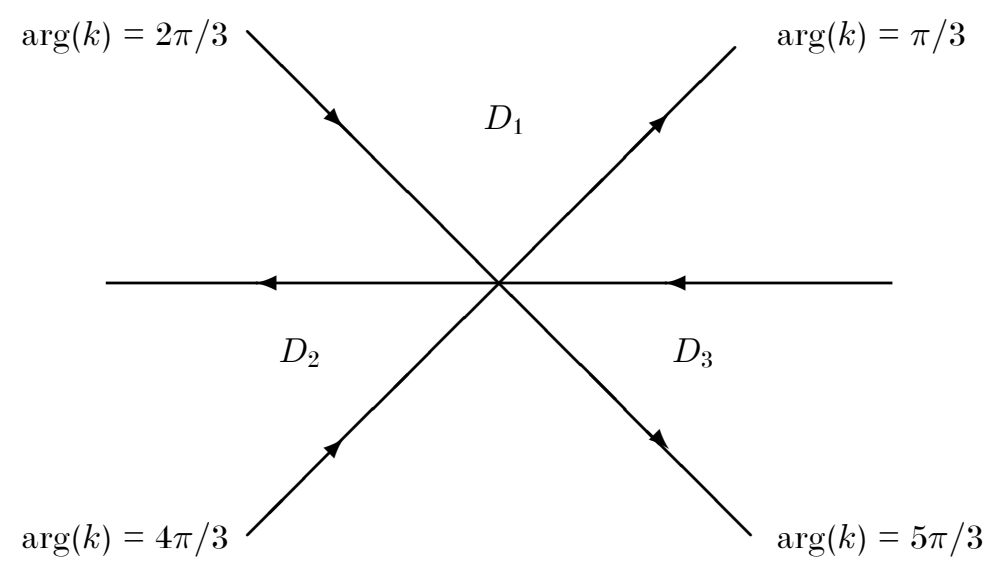

Fig. 2. The domains $D_{+}=D_{1}$ and $D_{-}=D_{2} \cup D_{3}$ for the equation $q_{t}+q_{x x x}=0$.

Thus $\omega(k)=-k^{3}$, and $D_{+}=D_{1}, D_{-}=D_{2} \cup D_{3}$ are the domains shown in Fig. 2 and defined by

$$
\begin{gathered}
D_{1}=\left\{k: \frac{\pi}{3} \leqslant \arg (k) \leqslant \frac{2 \pi}{3}\right\}, \\
D_{2}=\left\{k: \pi \leqslant \arg (k) \leqslant \frac{4 \pi}{3}\right\}, \quad D_{3}=\left\{k: \frac{5 \pi}{3} \leqslant \arg (k) \leqslant 2 \pi\right\} .
\end{gathered}
$$

The biholomorphic maps $\lambda_{l, m}(k)$ are given by

$$
\lambda_{1,1}(k)=k, \quad \lambda_{2,1}(k)=\zeta k, \quad \lambda_{3,1}(k)=\zeta^{2} k ; \quad \zeta=e^{-2 \pi i / 3} .
$$

A set of admissible functions with respect with the given initial data $q_{0}(x)=q(x, 0)$ contains six functions, $f_{0}(t), f_{1}(t), f_{2}(t), g_{0}(t), g_{1}(t)$ and $g_{2}(t)$. The spectral functions are given by

$$
\begin{aligned}
\hat{F}(k) & =\int_{0}^{T} e^{-i k^{3} t}\left[f_{2}(t)+i k f_{1}(t)-k^{2} f_{0}(t)\right] d t \\
& =\hat{f}_{2}(k)+k \hat{f}_{1}(k)-k^{2} \hat{f}_{0}(k), \\
\hat{G}(k) & =\int_{0}^{T} e^{-i k^{3} t}\left[g_{2}(t)-i k g_{1}(t)-k^{2} g_{0}(t)\right] d t \\
& =\hat{g}_{2}(k)+k \hat{g}_{1}(k)-k^{2} \hat{g}_{0}(k) .
\end{aligned}
$$

Hence for $k \in D_{1}$, the global relation yields

$$
\begin{aligned}
e^{i k L}\left[\hat{f}_{2}(k)+k \hat{f}_{1}(k)+k^{2} \hat{f}_{0}(k)\right]-\left[\hat{g}_{2}(k)+k \hat{g}_{1}(k)+k^{2} \hat{g}_{0}(k)\right] & =e^{i k L} \hat{q}_{0}(k)+e^{-i k^{3} T+i k L} \hat{a}(k), \\
{\left[\hat{f}_{2}(k)+\zeta k \hat{f}_{1}(k)+\zeta^{2} k^{2} \hat{f}_{0}(k)\right]-e^{-i \zeta k L} } & {\left[\hat{g}_{2}(k)+\zeta k \hat{g}_{1}(k)+\zeta^{2} k^{2} \hat{g}_{0}(k)\right] } \\
& =\hat{q}_{0}(\zeta k)+e^{-i k^{3} T} \hat{a}(\zeta k) \\
{\left[\hat{f}_{2}(k)+\zeta^{2} k \hat{f}_{1}(k)+\zeta k^{2} \hat{f}_{0}(k)\right]-e^{-i \zeta^{2} k L} } & {\left[\hat{g}_{2}(k)+\zeta^{2} k \hat{g}_{1}(k)+\zeta k^{2} \hat{g}_{0}(k)\right] } \\
& =\hat{q}_{0}\left(\zeta^{2} k\right)+e^{-i k^{3} T} \hat{a}\left(\zeta^{2} k\right) .
\end{aligned}
$$


This is a system of three equations for six unknowns. Assigning appropriately three boundary conditions, this becomes a system of three equations for three unknowns, admitting a unique solution. Although we have only proved this claim for the particular case that the boundary conditions $q(0, t), q(L, t)$ and $q_{x}(L, t)$ are assigned, the same holds true for other sets of boundary conditions, but not all set of three boundary conditions will yield a well defined system (see Example 5·2·2).

5.2.1 The boundary value problem for equation $(5 \cdot 15)$ defined by the initial and boundary conditions

$$
\begin{aligned}
q(x, 0) & =q_{0}(x), \quad 0<x<L, \\
q(0, t)=u_{0}(t), \quad q(L, t) & =v_{0}(t), \quad q_{x}(L, t)=v_{1}(t), \quad 0<t<T,
\end{aligned}
$$

where $q_{0}(x), u_{0}(t), v_{0}(t)$ and $v_{1}(t)$ are smooth functions, compatible at $x=t=0$ and at $x=L, t=0$, is well posed. The unique solution $q(x, t) \in \mathbf{C}^{3}[0, L] \times \mathbf{C}^{1}[0, T]$ is given by

$$
\begin{aligned}
q(x, t)= & \frac{1}{2 \pi}\left\{\int_{-\infty}^{\infty} e^{i k x+i k^{3} t} \hat{q}_{0}(k) d k\right. \\
& \left.+\int_{\partial D_{1}} e^{i k x+i k^{3} t}\left(-k^{2} \hat{u}_{0}(k)\right) d k+\int_{\partial D_{2} \cup \partial D_{3}} e^{i k(x-L)+i k^{3} t}\left(-k \hat{v}_{1}(k)-k^{2} \hat{v}_{0}(k)\right) d k\right\} \\
& +\int_{\partial D_{1}} e^{i k x+i k^{3} t} \frac{-k \tilde{B}_{1}(k)-\tilde{B}_{2}(k)}{2 \pi \Delta(k)} d k+\int_{\partial D_{2}} e^{i k(x-L)+i k^{3} t} \frac{-\tilde{B}_{3}(\zeta k)}{2 \pi \Delta(\zeta k)} d k \\
& +\int_{\partial D_{3}} e^{i k(x-L)+i k^{3} t} \frac{-\tilde{B}_{3}\left(\zeta^{2} k\right)}{2 \pi \Delta\left(\zeta^{2} k\right)} d k-i \sum_{h} e^{i k_{h}^{3} t} \frac{e^{i k_{h} x}\left(-k_{h} \tilde{B}_{1}\left(k_{h}\right)-\tilde{B}_{2}\left(k_{h}\right)\right)}{\Delta^{\prime}\left(k_{h}\right)} \\
& -i \sum_{h} e^{i k_{h}^{3} t} \frac{e^{i \zeta k_{h}(x-L)}\left(-\tilde{B}_{3}\left(k_{h}\right)\right)+e^{i \zeta^{2} k_{h}(x-L)}\left(-\tilde{B}_{3}\left(k_{h}\right)\right)}{\Delta^{\prime}\left(k_{h}\right)}
\end{aligned}
$$

where $\hat{q}_{0}(k)$ is defined by $(1 \cdot 9)$,

$$
\begin{gathered}
\hat{u}_{0}(k)=\int_{0}^{T} e^{-i k^{3} t} u_{0}(t) d t \\
\hat{v}_{0}(k)=\int_{0}^{T} e^{-i k^{3} t} v_{0}(t) d t, \quad \hat{v}_{1}(k)=\int_{0}^{T} e^{-i k^{3} t}\left(-i v_{1}(t)\right) d t,
\end{gathered}
$$

the functions $\tilde{B}_{1}(k), \tilde{B}_{2}(k)$ and $\tilde{B}_{3}(k)$ are explicitly expressed in terms of the given data, see equations $(5 \cdot 22)$ and $(5 \cdot 24)$, the determinant $\Delta(k)$ is given by

$$
\Delta=\frac{(\zeta-1) e^{i k L}}{\zeta^{2}} k\left(e^{-i k L}+\zeta^{2} e^{-i \zeta^{2} k L}+\zeta e^{-i \zeta k L}\right), \quad \zeta=e^{-2 \pi i / 3},
$$

$k_{h}$ are the zeros of $\Delta$ in the region $D_{1}$, and $\Delta^{\prime}(k)$ denotes the derivative with respect to $k$.

Indeed, let $E, E_{2}, E_{3}$ be defined by

$$
E=e^{i k L}, \quad E_{2}=e^{-i \zeta k L}, \quad E_{3}=e^{-i \zeta^{2} k L},
$$


with $\zeta$ given in $(5 \cdot 20)$. For $k \in D_{1}$, the system $(5 \cdot 16)$ can be used to define the unknown functions $\hat{f}_{1}(k), \hat{f}_{2}(k)$ and $\hat{g}_{2}(k)$ :

$$
\left(\begin{array}{ccc}
E k & E & -1 \\
\zeta k & 1 & -E_{2} \\
\zeta^{2} k & 1 & -E_{3}
\end{array}\right)\left(\begin{array}{c}
\hat{Q}_{1}(0, k) \\
\hat{Q}_{2}(0, k) \\
\hat{Q}_{2}(L, k)
\end{array}\right)=\left(\begin{array}{c}
b_{1} \\
b_{2} \\
b_{3}
\end{array}\right)+\left(\begin{array}{c}
a_{1} \\
a_{2} \\
a_{3}
\end{array}\right),
$$

where

$$
\left.\begin{array}{l}
b_{1}=E \hat{q}_{0}(k)-k^{2} E \hat{f}_{0}(k)+k^{2} \hat{g}_{0}(k)+k \hat{g}_{1}(k), \\
b_{2}=\hat{q}_{0}(\zeta k)-\zeta^{2} k^{2} \hat{f}_{0}(k)+E_{2}\left(\zeta^{2} k^{2} \hat{g}_{0}(k)+\zeta k \hat{g}_{1}(k)\right), \\
b_{3}=\hat{q}_{0}\left(\zeta^{2} k\right)-\zeta k^{2} \hat{f}_{0}(k)+E_{3}\left(\zeta k^{2} \hat{g}_{0}(k)+\zeta^{2} k \hat{g}_{1}(k)\right),
\end{array}\right\}
$$

and

$$
\left.\begin{array}{l}
a_{1}=e^{-i k^{3} T+i k L} \hat{a}(k), \\
a_{2}=e^{-i k^{3} T} \hat{a}(\zeta k), \\
a_{3}=e^{-i k^{3} T} \hat{a}\left(\zeta^{2} k\right) .
\end{array}\right\}
$$

The determinant $\Delta$ of this system is given by equation $(5 \cdot 20)$, and its unique solution is given by

$$
\begin{aligned}
\hat{Q}_{1}(0, k) & =\frac{\tilde{B}_{1}(k)}{\Delta(k)} \\
\tilde{B}_{1}(k) & =\left(b_{1}+a_{1}\right)\left(E_{2}-E_{3}\right)+\left(b_{2}+a_{2}\right)\left(E E_{3}-1\right)-\left(b_{3}+a_{3}\right)\left(E E_{2}-1\right), \\
\hat{Q}_{2}(0, k) & =\frac{\tilde{B}_{2}(k)}{\Delta(k)} \\
\tilde{B}_{2}(k) & \left.=\left(b_{1}+a_{1}\right) \zeta k\left(E_{3}-\zeta E_{2}\right)+b_{2}+a_{2}\right) k\left(\zeta^{2}-E E_{3}\right)+\left(b_{3}+a_{3}\right) k\left(E E_{2}-\zeta\right), \\
\hat{Q}_{2}(L, k) & =\frac{\tilde{B}_{3}(k)}{\Delta(k)} \\
\tilde{B}_{3}(k) & \left.=\left(b_{1}+a_{1}\right) \zeta k(1-\zeta)+b_{2}+a_{2}\right) E k\left(\zeta^{2}-1\right)-\left(b_{3}+a_{3}\right) E k(\zeta-1) .
\end{aligned}
$$

The determinant $\Delta(k)$ vanishes at $k=0$ and at the roots of the equation

$$
e^{-i k L}+\zeta^{2} e^{-i \zeta^{2} k L}+\zeta e^{-i \zeta k L}=0 .
$$

The convergence of the series corresponding to these zeros depends as before on the smoothness of the given data.

Remark $5 \cdot 2 \cdot 2$. It is noted in $[8]$ that if in Example $5 \cdot 2 \cdot 1$, the conditions $(5 \cdot 18)$ are replaced by

$$
q(0, t)=u_{0}(t), \quad q_{x}(0, t)=u_{1}(t), \quad q(L, t)=v_{0}(t), \quad 0<t<T,
$$

the resulting initial boundary value problem appears not to be well posed. This is due to the fact that, in this case, the determinant of the resulting system is such that $1 / \Delta$ is not bounded at infinity inside the region $D_{i}, i=1,2,3$; hence the system cannot be solved.

Acknowledgement. The authors were supported by EPSRC; they are grateful to L. Y. Sung for important suggestions. 


\section{REFERENCES}

[1] M.J. Ablowitz and A.S. Fokas. Introduction and applications of complex variables (Cambridge University Press, 1997).

[2] L. Cattabriga. Un problema al contorno per una equazione parabolica di ordine dispari. Annali della Scuola Norm. Sup. Pisa 13 (1959), 163-203.

[3] L. Ehrenpreis. Fourier analysis in several complex variables (Wiley-Interscience, 1970).

[4] A. S. Fokas. On the integrability of linear and nonlinear PDEs. J. Math. Phys. 41 (2000), $4188-4237$

[5] A. S. Fokas. Two dimensional linear PDE's in a convex polygon. Proc. Roy. Soc. Lond. A, 457 (2001), 371-393.

[6] A. S. Fokas and A. R. Its. The nonlinear Schrödinger equation on a finite interval, preprint, 2000.

[7] A. S. Fokas and L. Y. Sung. Initial boundary value problems for linear evolution equations on the half line, preprint, 1999.

[8] B. Pelloni. Two-point boundary value problems for integrable evolution equations in one space dimension. Annals of the MCFA 1 (1999), 30-35.

[9] E. C. Titchmarsh. Eigenfunction expansions associated with second order differential operators, Part I (Oxford University Press, 1955) 